\title{
Culture If Spikes? Indications and Yield of Blood Cultures in Hospitalized Medical Patients
}

\author{
Katherine Linsenmeyer, MD ${ }^{1,2 *}$, Kalpana Gupta, MD, MPH ${ }^{1,2}$, Judith M. Strymish, MD ${ }^{1,3}$, Muhammad Dhanani, MD ${ }^{1,2}$, \\ Stephen M. Brecher, $\mathrm{PHD}^{1,2}$, Anthony C. Breu, MD ${ }^{1,3}$
}

\begin{abstract}
${ }^{1}$ Veterans Affairs Boston Healthcare System, West Roxbury, Massachusetts; ${ }^{2}$ Boston University School of Medicine, Boston, Massachusetts; ${ }^{3}$ Harvard Medical School, Boston, Massachusetts.
\end{abstract}

BACKGROUND: Although optimal utilization of blood cultures has been studied in populations, including emergency room and intensive care patients, less is known about the use of blood cultures in populations consisting exclusively of patients on a medical service.

OBJECTIVE: To identify the physician-selected indication and yield of blood cultures ordered after hospitalization to an acute medical service and to identify populations in which blood cultures may not be necessary.

DESIGN, SETTING, AND PATIENTS: A prospective cohort study was performed at a single Veterans Affairs Medical Center from October 1, 2014 through April 15, 2015. Participants included all hospitalized patients on a medical service for whom a blood culture was ordered.

MEASUREMENTS: The main outcomes were the rate of true positive blood cultures and the predictors of true positive cultures.
RESULTS: The true positive rate was $3.6 \%$ per order. The most common physician-selected indications were fever and leukocytosis, neither of which alone was highly predictive of true positive blood cultures. The only indication significantly associated with a true positive blood culture was "follow-up previous positive" (likelihood ratio [LR]+ 3.4, $95 \%$ confidence interval [Cl]: 1.8-6.5). The only clinical predictors were a working diagnosis of bacteremia/ endocarditis (LR+3.7, 95\% Cl: 2.5-5.7) and absence of antibiotic exposure within 72 hours of the culture (LR+ 2.4 , 95\% Cl: 1.2-4.9).

CONCLUSIONS: The rate of true positive blood cultures among patients on a medical service was lower than previously studied. Using objective and easily obtainable clinical characteristics, including antibiotic exposure and working diagnosis, may improve the likelihood of true positive blood cultures. Journal of Hospital Medicine 2016;11:336-340. (C) 2016 Society of Hospital Medicine
Blood cultures are the gold standard test for the diagnosis of bloodstream infections (BSI). Given the high mortality associated with $\mathrm{BSI},{ }^{1-3}$ physicians have a low threshold to obtain blood cultures. ${ }^{4,5}$ Unfortunately, physicians are poor at predicting which hospitalized patients have BSI, ${ }^{6,7}$ and published guidelines do not provide clear indications for the use of blood cultures. ${ }^{8}$ As a result, current practice follows a "culture if spikes" paradigm, whereby inpatient providers often obtain blood cultures in the setting of any fever. This is the most common anticipatory guidance communicated between providers, involving up to $75 \%$ of written sign-out instructions. ${ }^{9}$ The result is a low rate of true positive blood cultures $(5 \%-10 \%)^{10-12}$ with only a slightly lower rate of false positive blood cultures (contaminants). ${ }^{12-14}$ False positive blood cultures often lead to repeat blood cultures, unnecessary antibiotic use, and increased hospital cost and length of stay. ${ }^{13}$

\footnotetext{
*Address for correspondence and reprint requests: Katherine Linsenmeyer, MD, Boston University School of Medicine, 850 Harrison Ave., Dowling Building 3rd Floor, Boston MA 02118; Telephone: 857-2035121; Fax: 857-203-5622; E-mail: katherine.linsenmeyer@bmc.org

Additional Supporting Information may be found in the online version of this article.

Received: October 7, 2015; Revised: December 7, 2015; Accepted: December 16, 2015

2016 Society of Hospital Medicine DOI 10.1002/jhm.2541

Published online in Wiley Online Library (Wileyonlinelibrary.com).
}

Over the last several years, there has been an increased emphasis on practicing high-value care by avoiding unnecessary and duplicate testing. In 2012, the American Board of Internal Medicine introduced the Choosing Wisely campaign, with specific initiatives to reduce medical waste and overuse. Given the low yield of blood cultures, guidance on patients in whom blood cultures are most appropriate would be welcome. Studies assessing risk factors for bacteremia have led to the development of multiple stratification systems without overall consensus. ${ }^{10,15-20}$ Furthermore, much of the current literature on blood culture utilization includes cultures drawn in the emergency department (ED) or intensive care unit setting (ICU). ${ }^{10,18-20}$ Less is known regarding the rates of positivity and utility for blood cultures drawn on patients hospitalized on an acute care medical ward.

Our study had 3 main objectives: (1) determine the rates of true positive and false positive blood cultures among hospitalized medical patients, (2) determine the ability of physician-selected indications and patient characteristics to predict BSI, and (3) identify populations in which blood cultures may not be necessary.

\section{PATIENTS AND METHODS Study Design}

We conducted a prospective cohort study of all hospitalized medical patients for whom blood cultures were 


\begin{tabular}{|c|c|c|c|}
\hline Clinical Characteristic & $\begin{array}{c}\text { Total, } \\
\mathrm{n}=363(\%)\end{array}$ & $\begin{array}{l}\text { True Positive Blood } \\
\text { Cultures, } n=14(\%)\end{array}$ & $P$ Value \\
\hline Mean age, y & 70.4 & 73.9 & 0.4 \\
\hline Male sex & $350(96 \%)$ & $14(100 \%)$ & 1 \\
\hline White race & $308(85 \%)$ & $11(79 \%)$ & 0.7 \\
\hline \multicolumn{4}{|l|}{ Location prior to admission } \\
\hline Community & $276(76 \%)$ & $11(79 \%)$ & 1 \\
\hline Hospital & $51(14 \%)$ & $1(7 \%)$ & 0.7 \\
\hline Long-term care facility & $36(10 \%)$ & $2(14 \%)$ & 0.6 \\
\hline \multicolumn{4}{|l|}{ Comorbidities } \\
\hline Diabetes & $136(37 \%)$ & $5(36 \%)$ & 1 \\
\hline Malignancy & $100(28 \%)$ & $4(31 \%)$ & 1 \\
\hline Alcohol abuse & $89(25 \%)$ & $2(14 \%)$ & 0.5 \\
\hline Cirrhosis & $31(9 \%)$ & $1(7 \%)$ & 1 \\
\hline End-stage renal disease & $21(6 \%)$ & $1(7 \%)$ & 1 \\
\hline Active drug use* & $16(4 \%)$ & $1(7 \%)$ & 0.5 \\
\hline Catheter $^{\dagger}$ & $93(26 \%)$ & $3(21 \%)$ & 0.8 \\
\hline Recent hospitalization ${ }^{\ddagger}$ & $145(40 \%)$ & $6(43 \%)$ & 1 \\
\hline History of MRSA colonization & $72(20 \%)$ & $5(36 \%)$ & 0.16 \\
\hline Cultures drawn in emergency department & $69(19 \%)$ & $6(43 \%)$ & 0.03 \\
\hline
\end{tabular}

NOTE: Abbreviations: MRSA, methicillin-resistant staphylococcus aureus. *Documented in admission note. ${ }^{\dagger}$ Includes urinary and central venous catheters. ${ }^{\ddagger}$ Within 90 days of current hospitalization.

ordered and received by the microbiology laboratory. This investigation was approved by the Veterans Affairs (VA) Boston Healthcare System internal review board.

\section{Patients and Setting}

During a 7-month period (October 1, 2014-April 15, 2015), all blood culture orders were reviewed for indication and result each day (and on Monday for weekend blood cultures) at a large VA teaching hospital (approximately 6200 admissions each year). As part of the electronic medical order, providers selected from among a list of common indications. Options included various clinical signs and diagnoses, and providers could select more than 1 indication. Each blood culture order triggered a phlebotomist to draw 2 separate blood culture sets (each set consisted of 1 aerobic and 1 anaerobic blood culture bottle).

Inclusion criteria included admission to 1 of 5 general medical service teams or 1 of 2 cardiology teams. Given that the study hospital does not have dedicated subspecialty service teams (with the exception of cardiology), all patients with medical diagnoses are cared for on the general medical service.

\section{Predictor and Outcome Variables}

Patient characteristics were obtained via chart review. Fever was defined as a single temperature greater than $100.4^{\circ} \mathrm{F}$ within 24 hours prior to a blood culture order. Leukocytosis was defined as a white blood cell count greater than 10,000 within 24 hours of a blood culture order. Patients were considered to have received antibiotics if an order for an antibacterial or antifungal agent was active within 72 hours prior to the blood culture order. Each blood culture order was assigned a working diagnosis that prompted the order. These working diagnoses were identified by chart review as documented under the provider's assessment and plan and were not necessarily the primary diagnosis prompting hospitalization.

Classification of positive blood cultures into true and false positive was determined by consensus among the microbiology and the infectious disease departments after review of clinical and laboratory data, consistent with a previously established practice at the hospital. A true negative culture consisted of any culture that was not a true positive or a false positive. A blood culture order was defined as an electronic entry and included all sets of blood cultures drawn as a result of that order. Consistent with previous literature, a blood culture episode was defined as all blood cultures ordered within a 48 -hour period starting at the time of the first culture. ${ }^{10}$ For patients with multiple admissions during the study period, each admission was considered a unique patient.

\section{Statistical Analysis}

Rates of true and false positivity of blood cultures were calculated. In addition, positive likelihood ratios $(\mathrm{LR}+)$ for true positive blood cultures were calculated using JMP statistical software (SAS Institute, Inc., Cary, NC).

\section{RESULTS \\ Overall}

A total of 576 blood culture orders ( 467 blood culture episodes) were completed on 363 hospitalized medical patients during the study period. Five hundred forty orders were placed on patients on general medical services and 36 orders on patients on the cardiology services. Four hundred eighty-seven $(85 \%)$ orders resulted in 2 sets of cultures being drawn, $87(15 \%)$ resulted in 1 set of cultures, and $2(0.3 \%)$ resulted in 3 sets of cultures. The median time between admission and culture draw was 2 days (range, 0-72 days), with $57 \%$ of cultures drawn during hospital day 0 to 2 , $24.5 \%$ drawn between hospital day 3 to 7 , and $19.4 \%$ drawn after hospital day 7. The average age of the patients was 70.4 years, and $94 \%$ were men. Additional patient characteristics are shown in Table 1.

The true positive and false positive rates per blood culture order were $3.6 \%$ (21/576) and $2.3 \%$ (13/576), respectively (Table 2 ). Similar values were seen per blood cultures episode $(3.4 \%$ and $2.7 \%$, respectively). The true positive blood culture rates per order and episode were significantly lower than those drawn on emergency room patients during the study period (41/570, 7.2\%, $P<0.05)$.

For the true positive cultures, gram-positive organisms were isolated most frequently $(14 / 21,67 \%)$ with Staphylococcus aureus identified in 2/21 (10\%) positive cultures and Enterococcus faecalis identified in 7/21 $(33 \%)$ positive cultures. Gram-negative organisms were 
TABLE 2. Rates of True Positive, False Positive, and True Negative Blood Cultures

\begin{tabular}{|c|c|c|c|c|}
\hline & Total, $\mathrm{n}(\%)$ & True Positive, n (\%) & False Positive, $n(\%)$ & True Negative, $\mathrm{n}(\%)$ \\
\hline Per patient & 363 & $14(3.8)$ & $13(3.6)$ & $336(92.6)$ \\
\hline Per blood culture episode & 467 & $16(3.4)$ & $13(2.7)$ & $438(93.8)$ \\
\hline Per blood culture order & 576 & $21(3.6)$ & $13(2.3)$ & $542(94.1)$ \\
\hline \multicolumn{5}{|l|}{ Rates per blood culture order } \\
\hline \multicolumn{5}{|l|}{ Physician-selected indication, $n=530$} \\
\hline Fever & $136(25.6)$ & $3(2.2)$ & $3(2.2)$ & $130(95.6)$ \\
\hline Fever and additional indication(s) & $118(22.2)$ & $5(4.2)$ & $3(2.5)$ & $110(93.2)$ \\
\hline Fever and leukocytosis & $50(9.4)^{\prime}$ & $4(8.0)$ & $3(6.0)$ & $43(86.0)$ \\
\hline Leukocytosis & $50(9.4)$ & $2(4.0)$ & $0(0)$ & $48(96.0)$ \\
\hline Follow-up previous positive & $60(11.3)$ & $7(11.7)$ & $0(0)$ & $53(88.3)$ \\
\hline \multicolumn{5}{|l|}{ Working diagnosis, $n=576$} \\
\hline Pneumonia & $101(17.5)$ & $0(0)$ & $4(3.9)$ & $97(96.0)$ \\
\hline Bacteremia/endocarditis & $97(16.8)$ & $12(12.3)$ & $1(1.0)$ & $84(86.6)$ \\
\hline Urinary tract infection* & $95(16.4)$ & $5(5.3)$ & $2(2.1)$ & $88(92.6)$ \\
\hline Other infection ${ }^{\dagger}$ & $46(8.0)$ & $0(0)$ & $0(0)$ & $46(100)$ \\
\hline Skin and soft-tissue infection & $39(6.8)$ & $1(2.6)$ & $0(0)$ & $38(97.4)$ \\
\hline Neutropenic fever & $28(4.9)$ & $0(0)$ & $0(0)$ & $28(100)$ \\
\hline Sepsis & $27(4.7)$ & $0(0)$ & $0(0)$ & $27(100)$ \\
\hline Fever $^{\ddagger}$ & $18(3.1)$ & $1(5.5)$ & $1(5.5)$ & $16(88.9)$ \\
\hline Bone and join infection & $15(2.6)$ & $1(6.7)$ & $0(0)$ & $14(93.3)$ \\
\hline Postoperative fever & $9(1.6)$ & $0(0)$ & $0(0)$ & $9(100)$ \\
\hline Noninfectious diagnosis ${ }^{S}$ & $101(17.5)$ & $1(1.0)$ & $5(5.0)$ & $95(94.1)$ \\
\hline \multicolumn{5}{|l|}{ Antibiotic exposure } \\
\hline Yes & $354(61.5)$ & $5(1.4)$ & $5(1.4)$ & $344(97.1)$ \\
\hline № & $222(38.6)$ & $16(7.2)$ & $8(3.6)$ & $198(89.1)$ \\
\hline \multicolumn{5}{|c|}{ Previous documented positive culture via chart review } \\
\hline Yes & $155(26.9)$ & $9(5.8)$ & $2(1.3)$ & $144(92.9)$ \\
\hline No & $421(73.1)$ & $12(2.9)$ & $11(2.6)$ & $398(94.5)$ \\
\hline
\end{tabular}

NOTE: *Includes pyelonephritis. IIncludes abdominal infections and meningitis. "Includes non-neutropenic and nonpostoperative fever. " Includes seizure, syncope, delirium, and heart failure.

isolated in $6 / 21(29 \%)$ cultures, and $1 / 21(5 \%)$ culture grew 2 organisms (Enterococcus faecalis and Nocardia). The majority of false positive cultures isolated 1

\begin{tabular}{|c|c|c|}
\hline & $\begin{array}{l}\mathrm{LR}+(95 \% \mathrm{Cl}) \text {, } \\
\text { True Positive } \\
\text { Blood Culture }\end{array}$ & $\begin{array}{l}\mathrm{LR}+(95 \% \mathrm{Cl}) \text {, } \\
\text { False Positive } \\
\text { Blood Culture }\end{array}$ \\
\hline \multicolumn{3}{|l|}{ Physician-selected indication } \\
\hline Fever & $0.6(0.2-1.7)$ & $0.9(0.3-2.5)$ \\
\hline Fever and additional indication(s) & $1.1(0.5-2.4)$ & $1.0(0.4-2.8)$ \\
\hline Fever and leukocytosis & $2.2(0.9-5.6)$ & $2.5(0.9-7.1)$ \\
\hline Leukocytosis & $1.1(0.3-4.0)$ & $0.4(0.0-5.6)$ \\
\hline Follow-up previous positive & $3.4(1.8-6.5)$ & $0.3(0.0-4.7)$ \\
\hline \multicolumn{3}{|l|}{ Diagnosis } \\
\hline Pneumonia & $0.1(0.0-1.9)$ & $1.8(0.8-4.1)$ \\
\hline Bacteremia/endocarditis & $3.7(2.5-5.7)$ & $0.5(0.1-3.0)$ \\
\hline Urinary tract infection & $1.5(0.7-3.2)$ & $0.9(0.3-3.4)$ \\
\hline Noninfectious diagnosis & $0.3(0.0-1.8)$ & $2.3(1.1-4.6)$ \\
\hline \multicolumn{3}{|l|}{ Recent antibiotic exposure } \\
\hline Yes & $0.4(0.2-0.8)$ & $0.6(0.3-1.2)$ \\
\hline No & $2.1(1.6-2.7)$ & $1.6(1.0-2.5)$ \\
\hline No with fever & $2.4(1.2-4.9)$ & $0.8(0.2-3.6)$ \\
\hline No with fever and leukocytosis & $5.6(1.8-18.2)$ & $0.4(0.1-2.6)$ \\
\hline \multicolumn{3}{|l|}{ Prior positive cultures } \\
\hline Yes & $1.6(1.0-2.7)$ & $0.6(0.2-2.0)$ \\
\hline
\end{tabular}

NOTE: Abbreviations: $\mathrm{Cl}$, confidence interval; $\mathrm{LR}+$, likelihood ratio positive. or more species of coagulase-negative Staphylococcus $(11 / 13,85 \%)$.

\section{Predictors of True Bacteremia}

The 4 most common working diagnoses prompting a blood culture order were pneumonia, bacteremia/endocarditis, urinary tract infection, and a noninfectious diagnosis (eg, syncope), with each prompting approximately $17 \%$ of the total orders (Table 2 ). Of these, only a primary diagnosis of bacteremia/endocarditis was predictive of a true positive culture, yielding a rate of $12.3 \%$ (LR $+3.7,95 \%$ confidence interval [CI]: 2.5 5.7). No other diagnosis was predictive of true positivity. A diagnosis of pneumonia yielded no true positive and 4 false positive blood cultures $(3.9 \%)$, whereas a noninfectious diagnosis yielded only 1 true positive $(1.0 \%)$ and 5 false positives $(5.0 \%)$. The positive likelihood ratios for these 2 diagnoses were 0.1 (95\% CI: $0.00-1.9)$ and 0.3 (95\% CI: 0.04-1.8), respectively.

Indications were selected for 530 of $576(92 \%)$ blood culture orders (Table 2). The most common indication was fever alone $(25.6 \%)$, followed by fever with an additional indication $(22.2 \%)$, follow-up positive blood cultures (11.3\%), fever and leukocytosis $(9.4 \%)$, and leukocytosis alone $(9.4 \%)$. Only followup positive blood cultures was predictive of a true positive, with a LR+ of 3.4 (95\% CI: 1.8-6.5). 
A total of 14 patients $(3.9 \%)$ had true positive blood cultures. For these patients, 10/14 (71\%) had 1 true positive blood culture, $3 / 14(21 \%)$ had 2 true positive blood cultures, and $1 / 14(7 \%)$ had 5 true positive blood cultures. The average number of cultures drawn was 4.9. The clinical characteristic most predictive of a true positive blood culture was the absence of recent antibiotic administration. If the blood culture was ordered on a patient not receiving antibiotics (true positivity rate $7.2 \%, 16 / 222$ ), the LR+ was 2.1 (95\% CI: 1.6-2.7). In a patient not receiving antibiotics who was also noted to have fever and leukocytosis (true positivity rate 17.6\%, 3/17), the LR+ was 5.6 (95\% CI: 1.8-18.2). Conversely, patients receiving antibiotics were rarely found to have true positive blood cultures (true positivity rate $1.4 \%, 5 / 354)$ with a LR+ of 0.4 (95\% CI: 0.2-0.8).

\section{DISCUSSION}

In this prospective study, we determined the diagnostic yield of blood cultures ordered on hospitalized medical patients to be low, with just $3.6 \%$ of orders identifying a true BSI. This was coupled with a similar false positive rate of $2.3 \%$. Our study found rates of true positive blood cultures much lower in hospitalized medical patients than in rates previously described when ED and ICU patients were included. ${ }^{11,16}$

Although ordering blood cultures is a routine clinical behavior when there is concern for an infection, a clinician's ability to subjectively predict who has a BSI only improves the likelihood 2-fold. ${ }^{6}$ Despite the availability of multiple scoring systems to aid the clinicians, ${ }^{10,21,22}$ our study found that over $50 \%$ of cultures were ordered in the setting of fever or leukocytosis, potentially demonstrating a triggered response to an event, rather than a decision based on probabilities. This common clinician instinct to "culture if spikes" is an ineffective practice if not coupled with additional clinical information. In fact, in 1 retrospective study, there was no association between fever spike and blood culture positivity. ${ }^{23}$

Our study suggests that objective and easily obtainable clinical characteristics may be effective in helping determine the probability of blood cultures revealing a BSI. Although more robust prediction models have value, they often require multiple inputs, limiting their utility to the bedside clinician. Stratifying patients by either antibiotic exposure or working diagnosis may provide the most benefit for the hospitalized medical patient. For those on antibiotics, the yield of true positive blood cultures is so low that they are unlikely to provide clinically useful information. In fact, although nearly two-thirds of cultures were obtained after antibiotic exposure, only $1(0.2 \%)$ of these patients had a culture that provided additional information regarding a BSI. Bacteremia had already been established for the other 4 patients. These results are similar to a prior study, which concluded that physicians should wait
72 hours from time of preantibiotic cultures before considering additional blood cultures given the lack of additional information provided. ${ }^{24}$

The working diagnosis also drives the probability of a positive blood culture. As has been shown with other studies, blood cultures are unlikely to diagnose a BSI for patients being treated for either cellulitis or pneumonia. ${ }^{25-27}$ In our study, the working diagnosis prompting the most blood cultures was pneumonia, with the false positive rate exceeding the true positive rate, a finding consistent with previous literature. This situation may lead to the addition of unnecessary antibiotics while waiting for a positive culture to be confirmed as a false positive (eg, vancomycin for a preliminary culture showing gram-positive cocci in clusters).

There are a number of limitations to our study. Physician-chosen indication may not correlate with the actual clinical picture and/or may not represent the full set of variables involved in the clinical decision to order a blood culture. However, the subjective clinical indication and the objective clinical criteria found in the chart provided similar LRs. Our study did not evaluate the potential harm of not ordering a blood culture. We also did not assess the value of a true negative culture particularly in patients with endovascular infections where additional cultures are often required to document clearance of bacteremia. Lastly, our study applies to patients on a hospitalized medical service and was performed at a VA hospital with a specific population of elderly male patients, which may limit the generalizability of our results.

Despite these limitations, this study benefits from its prospective design, along with the fact that $>90 \%$ of blood culture orders placed included a corresponding indication. This provides insight into physician clinical reasoning at the time the blood culture was ordered. In addition, our ability to calculate likelihood ratios provides bedside physicians with an easy and powerful way of modifying the probability of BSI prior to ordering blood cultures, aiding them in providing high-value clinical care while potentially reducing testing overuse.

The acceptability of not obtaining blood cultures may vary by clinical experience and by specialty. Physicians must weigh the low true positive rate against the consequences of missing a BSI. Although not a substitute for clinical judgement, the LRs in this study can provide a framework to aid in clinical decision making. For example, assuming a pretest probability of $3.6 \%$ (the rate of true positive for our entire cohort), blood cultures may not be equally as compelling in 2 similar patients with fever. The first is not on antibiotics and also has a leukocytosis. The second is being treated for pneumonia and is already on antibiotics. For the first patient, using a LR+ of 5.6 (for the fever and leukocytosis in the absence of antibiotics) modifies the patient's probability of a true positive blood culture to $17.3 \%$. Blood cultures should be ordered. In contrast, for the second patient, using a 
$\mathrm{LR}+$ of 0.4 (for the presence of antibiotics) decreases the patient's probability of a true positive blood culture to $1.5 \%$. Armed with these data, the bedside clinician can now decide whether this rate of true positivity warrants blood cultures. For some, this rate will be comfortably low. For others, this rate will not assuage them; only the negative culture will. Our data are not meant to make this decision, but may aid in making it a probabilitybased decision.

Disclosures: Presented in part at the Infectious Diseases Society of America Annual Meeting in San Diego, California in 2015. This material is the result of work supported in part with resources and the use of facilities at the VA Boston HCS, West Roxbury, MA. Katherine Linsenmeyer, MD, had full access to all of the data in the study and takes responsibility for the integrity of the data and the accuracy of the data analysis. The authors report no conflicts of interest.

\section{References}

1. Laupland KB, Church DL. Population-based epidemiology and microbiology of community-onset bloodstream infections. Clin Microbiol Rev. 2014;27(4):647-664

2. Weinstein MP, Towns ML, Quartey SM, et al. The clinical significance of positive blood cultures in the 1990s: a prospective comprehensive evaluation of the microbiology, epidemiology, and outcome of bacteremia and fungemia in adults. Clin Infect Dis. 1997;24(4): 584-602.

3. Weinstein MP, Murphy JR, Reller LB, Lichtenstein KA. The clinical significance of positive blood cultures: a comprehensive analysis of 500 episodes of bacteremia and fungemia in adults. II. Clinical observations, with special reference to factors influencing prognosis. Rev Infect Dis. 1983;5(1):54-70.

4. Kumar A, Roberts D, Wood KE, et al. Duration of hypotension before initiation of effective antimicrobial therapy is the critical determinant of survival in human septic shock. Crit Care Med. 2006;34(6): 1589-1596.

5. Sands KE, Bates DW, Lanken PN, et al. Epidemiology of sepsis syndrome in 8 academic medical centers. JAMA. 1997:278(3):234-240.

6. Pfitzenmeyer P, Decrey H, Auckenthaler R, Michel JP. Predicting bacteremia in older patients. J Am Geriatr Soc. 1995;43(3):230-235.

7. Makadon HJ, Bor D, Friedland G, Dasse P, Komaroff AL, Aronson MD. Febrile inpatients: house officers' use of blood cultures. J Gen Intern Med. 1987;2(5):293-297.

8. Baron EJ, Miller JM, Weinstein MP, et al. Executive summary: a guide to utilization of the microbiology laboratory for diagnosis of infectious diseases: 2013 recommendations by the Infectious Diseases Society of America (IDSA) and the American Society for Microbiology (ASM) (a). Clin Infect Dis. 2013;57(4):485-488.

9. Horwitz LI, Moin T, Krumholz HM, Wang L, Bradley EH. What are covering doctors told about their patients? Analysis of sign-out among internal medicine house staff. Qual Saf Health Care. 2009;18(4): $248-255$.

10. Bates DW, Cook EF, Goldman L, Lee TH. Predicting bacteremia in hospitalized patients. A prospectively validated model. Ann Intern Med. 1990;113(7):495-500.

11. Aronson MD, Bor DH. Blood cultures. Ann Intern Med. 1987;106(2): $246-253$.

12. Roth A, Wiklund AE, Palsson AS, et al. Reducing blood culture contamination by a simple informational intervention. J Clin Microbiol. 2010;48(12):4552-4558.

13. Bates DW, Goldman L, Lee TH. Contaminant blood cultures and resource utilization. The true consequences of false-positive results. JAMA. 1991;265(3):365-369.

14. Dawson S. Blood culture contaminants. J Hosp Infect. 2014;87(1): $1-10$.

15. Rangel-Frausto MS, Pittet D, Costigan M, Hwang T, Davis CS, Wenzel RP. The natural history of the systemic inflammatory response syndrome (SIRS). A prospective study. JAMA. 1995;273(2):117-123.

16. Bates DW, Sands K, Miller E, et al. Predicting bacteremia in patients with sepsis syndrome. Academic Medical Center Consortium Sepsis Project Working Group. J Infect Dis. 1997;176(6):1538-1551.

17. Jones GR, Lowes JA. The systemic inflammatory response syndrome as a predictor of bacteraemia and outcome from sepsis. QJM. 1996; 89(7):515-522.

18. Shapiro NI, Wolfe RE, Wright SB, Moore R, Bates DW. Who needs a blood culture? A prospectively derived and validated prediction rule. J Emerg Med. 2008;35(3):255-264.

19. Wildi K, Tschudin-Sutter S, Dell-Kuster S, Frei R, Bucher HC, Nuesch R. Factors associated with positive blood cultures in outpatients with suspected bacteremia. Eur J Clin Microbiol Infect Dis. 2011;30(12): $1615-1619$.

20. Yehezkelli Y, Subah S, Elhanan G, et al. Two rules for early prediction of bacteremia: testing in a university and a community hospital. $J$ Gen Intern Med. 1996;11(2):98-103.

21. Coburn B, Morris AM, Tomlinson G, Detsky AS. Does this adult patient with suspected bacteremia require blood cultures? JAMA. 2012;308(5):502-511.

22. Nakamura T, Takahashi O, Matsui K, et al. Clinical prediction rules for bacteremia and in-hospital death based on clinical data at the time of blood withdrawal for culture: an evaluation of their development and use. J Eval Clin Pract. 2006;12(6):692-703.

23. Riedel S, Bourbeau P, Swartz B, et al. Timing of specimen collection for blood cultures from febrile patients with bacteremia. J Clin Microbiol. 2008:46(4):1381-1385.

24. Grace CJ, Lieberman J, Pierce K, Littenberg B. Usefulness of blood culture for hospitalized patients who are receiving antibiotic therapy. Clin Infect Dis. 2001;32(11):1651-1655

25. Chalasani NP, Valdecanas MA, Gopal AK, McGowan JE Jr, Jurado RL. Clinical utility of blood cultures in adult patients with community-acquired pneumonia without defined underlying risks. Chest. 1995; 108(4):932-936.

26. Luna CM. Blood cultures in community-acquired pneumonia: are we ready to quit? Chest. 2003;123(4):977-978.

27. Craven DE. Blood cultures for community-acquired pneumonia: piecing together a mosaic for doing less. Am I Respir Crit Care Med. 2004;169(3):327-328. 